\title{
The electric double layer effect on the microchannel flow stability and heat transfer
}

\author{
Sedat Tardu* \\ LEGI, B.P. 53 X, 38041 Grenoble C'edex, France
}

The effect of the electric double layer (EDL) on the linear stability of Poiseuille planar channel flow is reported. It is shown that the EDL destabilises the linear modes, and that the critical Reynolds number decreases significantly when the thickness of the double layer becomes comparable with the height of the channel. First results coming from direct numerical simulations on the non-linear effects show also that the by-pass transition is much more rapid in the presence of EDL. There is an acceptable qualitative correspondence between the estimated transitional Reynolds numbers and some experiments, showing that early transition is plausible in microchannels under some conditions. Several questions remain however unanswered such as the surface conduction effect on EDL.

Keywords: Stability; Microchannels; Interfacial effects; Electric double layer

\section{Introduction}

The characteristics of gas flows in microchannels can adequately be modelled by slip velocity and temperature jump related to first or second order models as a function of the Knudsen number, at least in the rarefied regime. Such molecular effects are difficult to model in micro-liquid flows. Yet, estimations obtained from some molecular models indicate that the discontinuous boundary conditions could only hold for microchannels of hydraulic diameters smaller than a few microns [4, 23]. The interfacial effects (wall/liquid)

\footnotetext{
* Tel.: +33-4-76827030; fax: +33-4-76825271.

E-mail address: sedat.tardu@ @mg.inpg.fr (S. Tardu).
} 
are presumably mostly responsible for the deviations observed from the classical macrotheory, in larger microchannels. One of the micro-effects that may play an important role is the electric double layer (EDL) at the solid/liquid interface. The electrostatic charges present on the solid surface attract the counterions to establish an electrical field. In the compact layer next to the wall the ions are immobile. In the diffuse EDL layer however, the ions are less affected by the electrical field and can move. Under the effect of an imposed pressure gradient, the accumulation of the mobile ions downstream sets up an electrical field that induces a streamwise external force. In macroscale flows, these effects are negligible, as well as the thickness of the EDL is very small compared to the height of the channel. In micro-flows, in return, the EDL play a rather significant role. Well-controlled recent experiments have clearly confirmed that it can explain the behaviour of the Poiseuille number in the laminar regime, providing that the liquid contains a very small amount of ions [20]. Kulinsky et al. [11] reported an increase of $70 \%$ of the friction factor under the EDL effect in planar channels of $4 \mu \mathrm{m}$ heights with distilled water. Large thickness of the diffuse EDL layer of about $1 \mu \mathrm{m}$ or more have been reported in these experiments.

There is a curious phenomena encountered in some experiments showing that there is an early transition in microchannel flows. Wang and Peng [27] noticed that the transition occurred at $R e<150$ in microchannels $0.2-0.8 \mathrm{~mm}$ wide and $0.7 \mathrm{~mm}$ deep, which is significantly smaller than 400 corresponding to macroscale flows. (The Reynolds number through this paper is based on the centreline velocity and half height of the channel. Thus 400 corresponds to a Reynolds number based on hydraulic diameter and cross section average velocity of 2000) A transitional number of about 250 has also been reported in the experiments conducted in $1 \mathrm{~mm}$ wide trapezoidal microchannels with channel depths ranging between 79 and $325 \mu \mathrm{m}[7,18,19]$. The microtubes experiments of [15] indicate that there is an early transition from laminar to turbulent flows for $R e>56-170$. These effects may be attributed to the roughness that may both influence the transition in the entry region, or directly affect the flow behaviour through an implied roughness-viscosity [28]. Yet, direct interfacial effects may also play a role in presumed early transition.

The stability mechanism in planar channel flows is mainly non-linear and the secondary instabilities cause the flow to bifurcate before the critical Reynolds number of linear modes. That depends on the behaviour of the time space development of the perturbations near the critical $R e$ and wave numbers. The main aim of this study is to investigate directly the EDL effect on the linear stability of planar channel flow, and estimate indirectly the resulting transitional Reynolds numbers. It is asked whether electrokinetic effects on liquid flows may cause the early transition or not and whether the linear stability EDL flow characteristics may explain the small transitional Reynolds numbers reported so far in some experiments.

\section{Physical aspects of electric double layers}

Most solid surfaces have an electric surface potential when brought within an electrolyte. The most common mechanism for the charging of surface layers in microfluidics is the deprotonation of surface groups on surfaces such as silica, glass, acrylic and polyester $[9,22]$. The electrostatic charges present on the solid surface attract the 


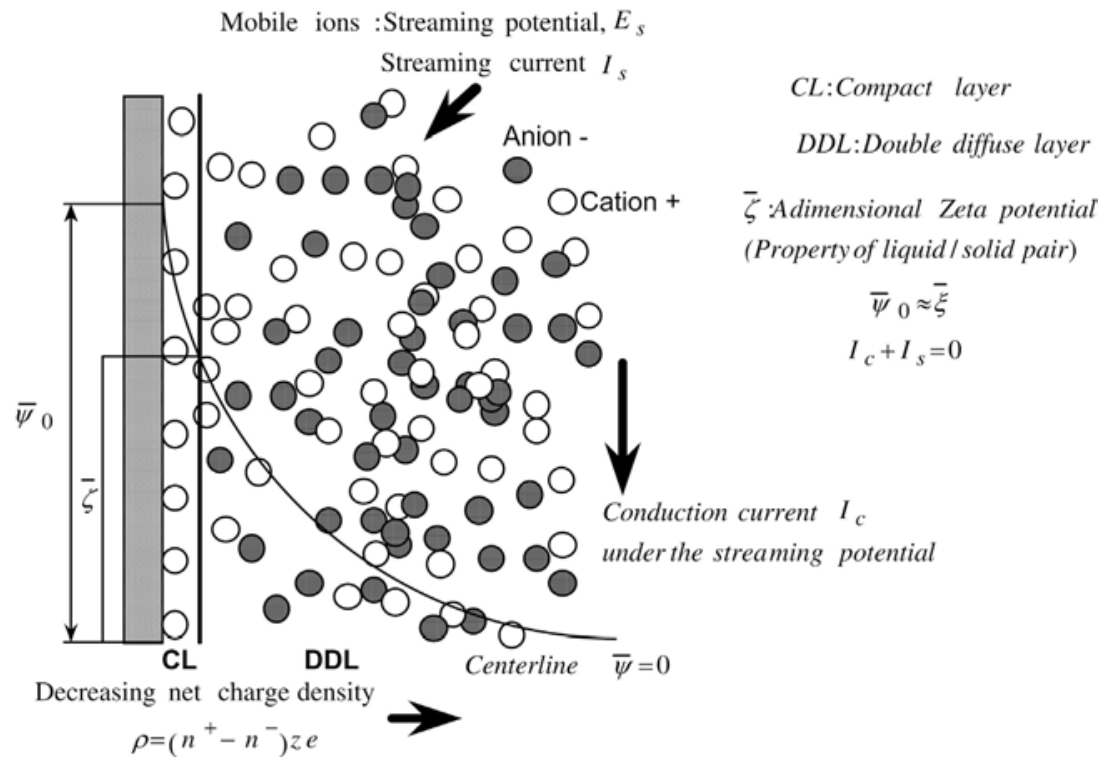

Fig. 1. EDL effect.

counterions to establish an electrical field (Fig. 1). In the compact layer next to the wall and less than $1 \mathrm{~nm}$ thick the ions are immobile. In the diffuse EDL layer however, the ions are less affected by the electrical field and can move. The counterion concentration near the wall is larger than in the bulk of the fluid. That results in a net charge density in a unit volume resulting from the concentration difference between cations and anions, according to the Boltzmann equation. The electrostatic potential at any point near the surface, provided that it is small compared to the energy of ions, may be obtained by a linear approximation of the Poisson-Boltzmann equation. Its value at the wall can be related to the Zeta potential between the compact layer and diffuse layer, when the EDLs near the opposite walls do not overlap. The Zeta potential is a property of the solid-liquid pair and can be determined experimentally. The imposed pressure gradient accumulates the mobile ions downstream and sets up an electrical field whose potential is called the streaming or electrokinetic potential. The streaming potential and the net charge density induces a streamwise external force. In the steady state, the streaming current due to the transport of charges is in equilibrium with the conduction current in the opposite direction. That allows the determination of the streaming potential and of the velocity profiles under the EDL effect. In macroscale flows, these effects are negligible, as well as the thickness of the EDL is very small compared to the height of the channel.

\section{Equations governing 2D channel flow under the EDL effect}

We suppose constant properties (viscosity and permittivity). The effects of finite ion size and gradients of the dielectric strength and of the viscosity are neglected. These hypotheses are not contradictory with the fact that we mainly deal with very dilute solutions for which 
the equilibrium Boltzmann distribution is applicable. Thus, the number of ion distribution in a symmetric plane channel flow is given by

$$
n^{ \pm}=n_{0} \exp \left(\mp \frac{z e \psi}{k_{\mathrm{B}} T}\right)
$$

where $n_{0}$ is the bulk ionic concentration, $\psi$ is the electrical potential, $z$ is the valence of positive or negative ions, $e$ is the electron charge, $k_{\mathrm{B}}$ is the Boltzmann constant and $T$ is the absolute temperature. The resulting net charge density in a unit volume of fluid is

$$
\rho=\left(n^{+}-n^{-}\right) z e=-2 n_{0} \sinh \left(\frac{z e \psi}{k_{\mathrm{B}} T}\right) .
$$

The Poisson equation relating the electrical potential and the net charge density per unit volume reads as:

$$
\nabla^{2} \psi=-\frac{\rho}{\varepsilon_{0} \varepsilon}
$$

with $\varepsilon$ and $\varepsilon_{0}$ standing respectively for the dielectric constant of the medium and the permittivity of vacuum. Eq. (3) combined with (2) may be written in non-dimensional form as

$$
\nabla^{2} \bar{\psi}=-\kappa^{2} \bar{\rho}(y)=\kappa^{2} \sinh (\bar{\psi}) .
$$

The scaling reference potential and charge density are respectively $E_{S}=k_{\mathrm{B}} T / z e$ and $\rho_{S}=n_{0} z e$. Eq. (4) makes the non-dimensional Debye parameter $\kappa=k a$ appear with:

$$
k=\left(2 n_{0} z^{2} e^{2} / \varepsilon \varepsilon_{0} k_{\mathrm{B}} T\right)^{1 / 2}
$$

standing for the Debye-Huckel parameter whose inverse is the EDL thickness, and $a$ is the channel half height.

Eq. (4) may be linearized when the electrical potential is small compared with the thermal energy of ions, i.e. when $|z e \psi| \ll\left|k_{\mathrm{B}} T\right|$, or $\bar{\psi} \ll 1$ to give:

$$
\nabla^{2} \bar{\psi}=\kappa^{2} \bar{\psi}
$$

which holds remarkably well for $\bar{\psi}<4$. This value is equivalent to a Zeta potential of roughly $100 \mathrm{mV}$ which is within the typical range in microfluidics applications [22], even though values as large as $\zeta=245 \mathrm{mV}$ are reported in the literature [20]. For a twodimensional channel, Eq. (6) reduces to:

$$
\frac{\mathrm{d}^{2} \bar{\psi}}{\mathrm{d} y^{2}}=\kappa^{2} \bar{\psi}
$$

with $y=Y / a$, the non-dimensional wall normal distance. The charge density is then simply $\bar{\rho}(y)=-\bar{\psi}=-\left(1 / \kappa^{2}\right) /\left(\mathrm{d}^{2} \bar{\psi} / \mathrm{d} y^{2}\right)$. The boundary conditions for Eq. (7) are:

- At the wall $\psi=\bar{\psi}_{0}$. The electrical potential at the wall can be approximated by the Zeta potential $\bar{\psi}_{0}=\bar{\zeta}$, when the electrical charge of the surface is small. The general solution of the resulting EDL-velocity profile is not affected by this approximation. However, it is generally difficult to measure $\bar{\psi}_{0}$. It is much easier to determine experimentally the Zeta potential at the boundary between the compact layer and diffuse layer, by determining the streaming potential and the pressure drop [13]. 
- At the centreline, $\bar{\psi}=0$, a condition which is valid typically for $\kappa \geq 2$, providing that the EDL diffuse layers developing on both sides of the channel do not overlap.

The solution of (7) can easily be obtained and is given by:

$$
\bar{\psi}=\frac{\bar{\zeta}}{\sinh (\kappa)}|\sinh (\kappa y)| .
$$

The charge density $\bar{\rho}(y)$ and the streaming electrical field strength caused by the ions mobilized under the imposed pressure gradient acts as an external force in the streamwise momentum equation of a fully developed 2D channel flow:

$$
0=\mu \frac{\mathrm{d}^{2} U}{\mathrm{~d} Y^{2}}-\frac{\mathrm{d} P}{\mathrm{~d} X}+E_{x} \rho(Y) .
$$

The electrical field strength is related to the streaming potential by $E_{x}=E_{s} / l$, where $l$ is the length of the channel. The non-dimensional form of (9) is:

$$
0=\frac{\mathrm{d}^{2} u}{\mathrm{~d} Y^{2}}+2-\frac{2 \Gamma \bar{E}_{s}}{\kappa^{2}} \frac{\mathrm{d}^{2} \bar{\psi}}{\mathrm{d} y^{2}}
$$

where $\Gamma=\zeta n_{0} z e a^{2} / l \mu U_{s}$ and the scaling velocity is the centreline velocity of the Poiseuille component, i.e. $U_{s}=-\left(a^{2} \mathrm{~d} p / \mathrm{d} x\right) / 2 \mu$. Integrating (10) with appropriate boundary conditions gives the velocity profile:

$$
u=1-y^{2}-4 \frac{\Gamma \bar{E}_{s} \bar{\zeta}}{\kappa^{2}}\left\{1-\left|\frac{\sinh \kappa y}{\sinh \kappa}\right|\right\} .
$$

The last equation is the non-dimensional form of the velocity profile already obtained by [14]. The streaming current due to the flux of the net density charge is given by the following cross integral by definition:

$$
I_{s}=\iint U \rho(Y) \mathrm{d} Y \mathrm{~d} Z .
$$

The streaming current produces the conduction current in the reverse direction:

$$
I_{c}=E_{x} \lambda_{0} S=\frac{E_{s}}{l} \lambda_{0} S
$$

where $\lambda_{0}=\lambda_{b}+\lambda_{s} / a$ is the total electrical conductivity of the fluid, with $\lambda_{b}$ standing for the bulk electrical conductivity which is proportional to the ionic concentration and $\lambda_{s}$ is the surface conductance due to the charged solid-liquid interface [3]. In the steady state there is no net current $I_{S}+I_{c}=0$. This allows the closure of the equations and the determination of the streaming potential. The final version of the non-dimensional velocity profile is:

$$
u=1-y^{2}-4 \frac{I_{1}-I_{2}}{\frac{\kappa^{2} \sinh \kappa}{\bar{\zeta}^{2} G}+4\left(I_{3}-\frac{I_{4}}{\sinh \kappa}\right)}\left\{1-\left|\frac{\sinh \kappa y}{\sinh \kappa}\right|\right\} .
$$


The parameter $G$ is given by $G=\left(n_{0} z e a\right)^{2} /\left(\lambda_{0} \mu\right)$. The quantities $I$ in (1) are: ${ }^{1}$

$$
\begin{aligned}
& I_{1}=I_{3}=\frac{\cosh \kappa}{\kappa} \\
& I_{2}=\left(\frac{1}{\kappa}+\frac{2}{\kappa^{3}}\right) \cosh \kappa-\frac{2}{\kappa^{2}} \sinh \kappa-\frac{2}{\kappa^{3}} \\
& I_{4}=\frac{\sinh \kappa \cosh \kappa}{2 \kappa}-\frac{1}{2} .
\end{aligned}
$$

It is seen that the velocity profile can be decomposed into a macro-Poiseuille component plus an EDL effect component. A closer inspection of (14) allows expressing the latter in another close form as:

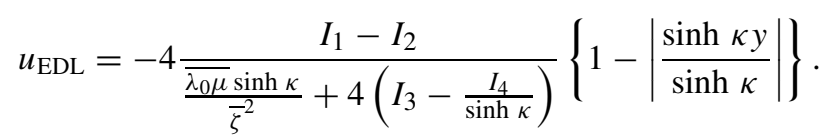

The parameter $\overline{\lambda_{0} \mu}$ is the non-dimensional electrical conductivity times the dynamic viscosity of the fluid. It can be seen that the scaling parameter for $\lambda_{0} \mu$ is the square of the scaling charge density times the scaling length, i.e. $\left(\rho_{S} / k\right)^{2}$ leading to:

$$
\overline{\lambda_{0} \mu}=\frac{\lambda_{0} \mu}{\left(n_{0} \varepsilon \varepsilon_{0} k_{\mathrm{B}} T / 2\right)} .
$$

By neglecting the surface conductance effect, the streaming potential can be written as [12]:

$$
\frac{E_{s}}{\Delta P}=\frac{\varepsilon \varepsilon_{0 \zeta}}{\mu \lambda_{b}} \frac{J_{1}}{1+\frac{J_{2}\left(\kappa \varepsilon \varepsilon_{0 \zeta}\right)^{2}}{\sinh ^{2}(\kappa) a^{2} \mu \lambda_{0}}}
$$

with $\Delta P=-(\mathrm{d} p / \mathrm{d} x) l, l$ being the length of the channel and $J_{1}=\left(\kappa^{2} / 2 \sinh (\kappa)\right)$ $\left(I_{1}-I_{2}\right), J_{2}=I_{3} \sinh (\kappa)-I_{4}$. The first group of terms in (18) is the classical Helmholtz-Smoluchowski equation, and the second is the correction coefficient coming from the EDL and surface conductance effects.

There are three parameters governing the EDL effect, namely

$$
u_{\mathrm{EDL}}=u_{\mathrm{EDL}}\left(\kappa, \bar{\zeta}, \overline{\lambda_{0} \mu}\right) .
$$

Gradients of the dielectric strength, viscosity and conductivity should be incorporated in more realistic models. Yet, these effects are presumably negligible in significantly dilute solutions and pure organic liquids.

The main EDL effects may be summarized as follows:

- An increase of the friction constant and apparent viscosity.

- A decrease of the Nusselt number.

These effects are persistent yet not significantly important at least for large values of $\kappa$. The apparent viscosity increases for example by a factor of nearly 3 at $\kappa=2$, but the EDL effect

\footnotetext{
${ }^{1}$ There is a small printing error in [14, p. 3082].
} 
on the wall shear stress disappears quickly when $\kappa \geq 10$ even at somewhat unacceptably large $G \bar{\zeta}^{2}$ values [14]. The Nusselt number decreases by nearly $40 \%$ at $\kappa=5$, and less than $5 \%$ at $\kappa=50$ as it will be discussed in the next section [23].

\section{Physical parameters and discussion}

The electrokinetic effects are significant for respectively small and large values of the parameters $\kappa$ and $G \bar{\zeta}^{2}$. The first numerical investigations related to the EDL in microchannel flows used severe parameters to probe on the importance of the EDL effects. Thus Mala et al. [14] took an infinitely diluted aqueous ideal $1: 1 \mathrm{KCl}$ solution in a $25 \mu \mathrm{m}$ height, $1 \mathrm{~cm}$ long channel with a pressure difference of 4 ATM, $n_{0}=6.022 \times 10^{20} \mathrm{~m}^{-3}$. To illustrate their theoretical work, they further assumed that the surface conductance is negligible with $\lambda_{0} \approx \lambda_{b}=1.2639 \times 10^{-7} \mathrm{~S} \mathrm{~m}^{-1}$, and that the Zeta potential is $50 \mathrm{mV}$. More severe cases have been analysed in the literature: Yang et al. [29] give numerical results using the non-linear Poisson-Boltzman equation for a (hypothetical) $1: 1$ $\mathrm{KCl}$ ideal electrolyte with $n_{0}=6.022 \times 10^{18} \mathrm{~m}^{-3}, \lambda_{0}=1.2639 \times 10^{-9} \mathrm{~S} \mathrm{~m}^{-1}$ in a $100 \mu \mathrm{m}$ hydraulic diameter 2D channel subject to a $200 \mathrm{mV}$ Zeta potential, giving raise to $G \bar{\zeta}^{2}=158 \times 10^{3}$, and $\kappa=16$ ! The careful reader could notice that such low conductivities and high-pressure differences $\Delta P$ would lead to unrealistically large streaming potentials according to Eq. (8). In reality the surface conductance term $\lambda_{s} / a$ is much larger than $\lambda_{b}$ in microchannels. Thus the real streaming potential is several orders of magnitude smaller and leads consequently to a weaker EDL effect.

Other liquid/surface configurations would certainly be more convenient for experimental purposes. Note that the aim here is to reach significant electro-viscous effects, contrarily to electro-osmotic flows. Thus, dielectric liquids with low conductivity would be convenient. One could for instance use some hydrocarbons combined with additives. Such liquids have been largely investigated in the literature for a while for the electrification problems. The Debye length in a cyclohexane $\left(\mathrm{C}_{6} \mathrm{H}_{12}\right)$ flow containing the tri isoamylammonium picrate is for example as large as $40 \mu \mathrm{m}[1, \mathrm{p} .51]$ and the Zeta potential is $\zeta=45 \mathrm{mV}$.

We consider a cyclohexane flow containing 2-ethylhexyl sulfosuccinate sodium. This additive may vary the conductivity of the base fluid from roughly $10^{-11}$ to $10^{-8} \mathrm{~S} \mathrm{~m}^{-1}$. The relative dielectric constant of the medium is $\varepsilon=2$ and $\mu=10^{-3} \mathrm{~kg} \mathrm{~m} / \mathrm{s}$. The Debye length is $1 / k=12 \mu \mathrm{m}$ at $\lambda_{b}=3 \times 10^{-11}$. A minichannel of $1 \mathrm{~mm}$ height would be sufficient to achieve $\kappa=41$. The pressure difference necessary to reach a Reynolds number of $10^{3}$ in a $1 \mathrm{~mm}$ height and $1 \mathrm{~cm}$ long minichannel is $\Delta P=160 \mathrm{~Pa}$. The Zeta potential is about $\zeta=100 \mathrm{mV}$ for which the linear approximation of the Boltzmann equation still gives satisfactory results according to Yang et al. [30].

Unfortunately we are not aware of the experimental values of the surface conductance corresponding to this case. We know however that as the ionic concentration decreases the surface conductance decreases too, unlike the Zeta potential. Ren et al. [20, 21] reported that $\lambda_{s}=10^{-7} \mathrm{~S}$ for deionized water with $\lambda_{b}=10^{-4} \mathrm{~S} \mathrm{~m}^{-1}$ for a $40 \mu \mathrm{m}$ height channel. The surface conductance increases to $\lambda_{s}=7 \times 10^{-7} \mathrm{~S}$ with $\lambda_{b}=15 \times 10^{-4} \mathrm{~S} \mathrm{~m}^{-1}$ corresponding to an $10^{-4} \mathrm{M} \mathrm{KCl}$ solution. The channel height affects also $\lambda_{s}$ that increases 
roughly linearly with $a$. By extrapolating the same qualitative behaviour to our case, one finds $\lambda_{s} \approx 4 \times 10^{-13} \mathrm{~S}$ for the cyclohexane solution and $\lambda_{s} / a \approx 8 \times 10^{-10} \mathrm{~S} \mathrm{~m}^{-1}$. The resulting non-dimensional parameters $G$ and $\bar{\zeta}$ are respectively $G=3000$ and $\bar{\zeta}=4$. This case will be labelled A hereafter.

It has to be emphasised here that the role played by the surface conductance in the electro-viscous flows is not clearly established yet and inconsistencies persist in some recent studies. There are for example some contradictions in the well-controlled experiments conducted by Ren et al. [20] as discussed in detail by these authors. As we have already mentioned the surface conductance term $\lambda_{s} / a$ becomes predominant in microchannels and may reach large values. However, Ren et al. [20] have noticed that the model prediction (Eq. (7)) agrees well with the experiments when the surface conductance is not considered and suggested revisiting in the future the way $\lambda_{s}$ is introduced in the electrokinetic flow model. But, if $\lambda_{s}$ is not considered in their experiments, the streaming potential they should measure should increase by a factor of 50 and be about $15 \mathrm{~V}$ at $R e \approx 50$ in a $40.5 \mu \mathrm{m}$ channel. The streaming potential corresponding to the case A investigated here is also large $E_{s} \approx 100 \mathrm{~V}$ but at a larger $R e \approx 1000$. Considering the crudeness of the approximation made to establish the case A, we will also discuss the cases $\mathrm{B}$ and $\mathrm{C}$ with respectively $G=300\left(E_{s} \approx 10 \mathrm{~V}\right)$ and $G=30\left(E_{s} \approx 1 \mathrm{~V}\right)$, corresponding to conductances that are ten and one hundred times larger. An intermediate reference case D with $G=600$ will be discussed in the session related to the by-pass transition. In all the cases, $\bar{\zeta}=4$ and $\kappa=41$ in the generic $2 a=1 \mathrm{~mm}$ channel, the fluid is the same and the $\kappa$ variations are only due to the modifications of the channel width. The most physically reasonable cases are, in order, $\mathrm{C}$ and $\mathrm{B}$, but we keep also the cases $\mathrm{A}$ and $\mathrm{D}$ to probe to strong EDL effects.

\section{EDL effect on heat transfer}

It is possible to obtain exact analytical solutions concerning the effect of EDL on the Nusselt number subject to constant heat flux. It is supposed that the flow is hydraulically and thermally developed, the Péclet number $P e=R e P r$ is large and the ratio of the Eckert to the Reynolds numbers $E c / R e$ is small so that the axial conduction and the dissipation are negligible. It is well known that in such case the Nusselt number is given by:

$$
N u=\left\{\int_{0}^{1} \frac{u}{u_{V}}\left[\int_{\eta}^{1} \int_{0}^{\eta^{\prime}} \frac{u}{u_{V}} \mathrm{~d} \eta^{\prime \prime} \mathrm{d} \eta^{\prime}\right] \mathrm{d} \eta\right\}^{-1} .
$$

The Nusselt number in (19) is based on the half channel height and the bulk temperature. The cross sectional average bulk velocity is denoted by $u_{V}$ :

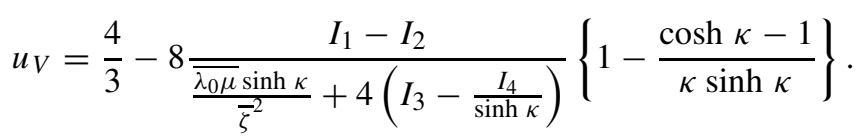

Eqs. (14) and (19) can be combined to obtain the Nusselt number variation versus the parameters involved in the problem. The computation is not difficult but it is long and tedious. The result will be given here in the form: 


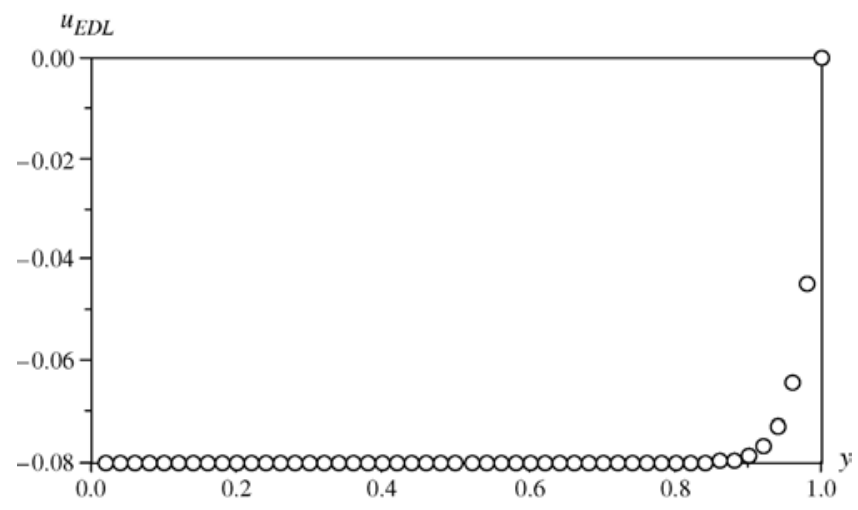

Fig. 2. The velocity distribution of the EDL flow component in the half channel cross section for the reference case A (see the text).

$$
N u^{-1}=S_{1}+S_{2}+S_{3}
$$

where one has respectively:

$$
\begin{aligned}
S_{1}= & (\alpha-\beta)\left\{\frac{4 \alpha}{15}+\beta\left[-\frac{1}{3}-\frac{1}{\kappa \sinh \kappa}+\frac{1}{\kappa^{2}}-\frac{\cosh \kappa-1}{\kappa^{3} \sinh \kappa}\right]\right\} \\
S_{2}= & \alpha\left\{\left(\frac{1}{63}-\frac{1}{15}\right) \alpha+\beta\left[\frac{1}{15}+\frac{1}{12 \kappa \sinh \kappa}-\frac{1}{3 \kappa^{2}}\right.\right. \\
& \left.\left.+\frac{1}{\kappa^{5} \sinh \kappa}\left[\left(2+\kappa^{2}\right) \cosh \kappa-2 \kappa \sinh \kappa-2\right]\right]\right\} \\
S_{3}= & \frac{\beta}{\sinh \kappa}\left\{\frac{\sinh \kappa}{\kappa^{3}}\left[\frac{7}{6} \alpha-\left(1+\frac{1}{\sinh \kappa}\right) \beta\right]\right. \\
& +\cosh \kappa\left[\frac{1}{12}-\frac{1}{6 \kappa}-\frac{1}{12 \kappa^{2}}-\frac{2+\beta}{\kappa^{3}}-\frac{1}{16 \kappa^{4}}\right] \\
& \left.+\alpha\left[-\frac{5}{12 \kappa}+\frac{1}{\kappa^{3}}+\frac{1}{6 \kappa^{4}}\right]+\beta\left[\frac{1}{2 \kappa}+\frac{1}{2 \kappa^{2} \sinh \kappa}-\frac{2}{\kappa^{3}}\right]\right\} .
\end{aligned}
$$

In these expressions the parameters $\alpha$ and $\beta$ are respectively:

$$
\begin{aligned}
\alpha & =\frac{1}{u_{V}} \\
\beta & =\frac{4}{u_{V}} \frac{I_{1}-I_{2}}{\frac{\kappa^{2} \sinh \kappa}{\bar{\zeta}^{2} G}+4\left(I_{3}-\frac{I_{4}}{\sinh \kappa}\right)} .
\end{aligned}
$$

These relationships are complicated. It is however possible to give simpler forms for large values of the Debye lengths, say typically $\kappa \geq 10$. The EDL component is then constant over a large part of the channel section and may be considered as a plug flow. Fig. 2 shows the $u_{\mathrm{EDL}}$ distribution for the case A. It is seen that $u_{\mathrm{EDL}}$ is constant over $90 \%$ of the cross section. Neglecting the contribution of the variations in $u_{\mathrm{EDL}}$ and assuming 


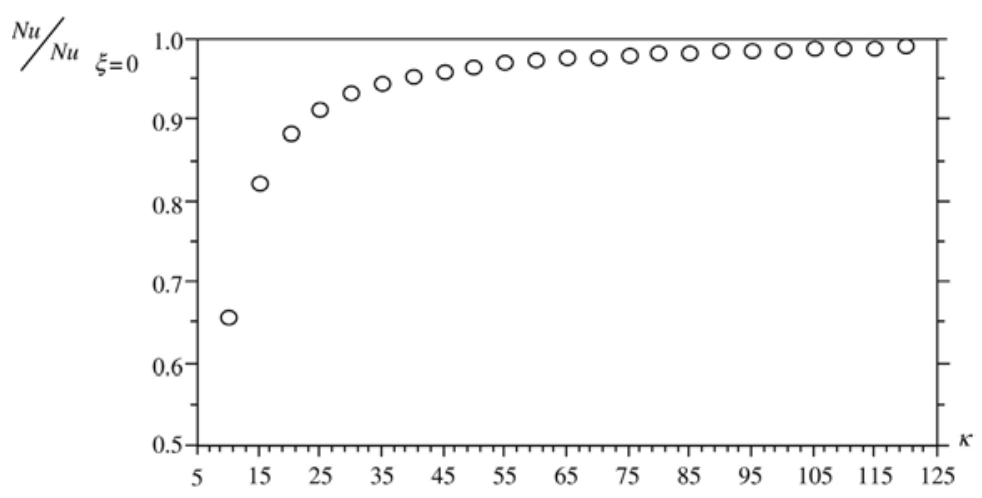

Fig. 3. Ratio of the Nusselt number in a channel flow with constant heat flux with and without EDL effect for the reference flow A (see the text).

that it is equal to its core value everywhere one obtains:

$$
N u=\frac{h a}{k}=\frac{\partial}{\partial(y / a)_{p}} \frac{T_{w}-T}{T_{w}-T_{V}}=\frac{(1+3 r / 2)^{2}}{\frac{17}{35}+\frac{6}{5} r+\frac{3 r^{2}}{4}}
$$

where $a$ is the half height of the channel, $T_{w}$ and $T_{V}$ are respectively the wall and bulk temperatures. This relationship is a good approximation, which is valid typically for $\kappa \geq 10$. The reader is referred to [23] for further details. The coefficient $r$ in (3) is the ratio of the EDL to the Poiseuille velocity components at the centreline that reads as:

$$
r=\frac{U_{\mathrm{EDL}}(y=0)}{U_{P}(y=0)} \cong-\frac{8 G \bar{\xi}^{2}}{\kappa\left(\kappa^{3}+2 G \bar{\xi}^{2}\right)}
$$

and $r \leq 0$. Fig. 3 shows the variations of the Nusselt number versus the non-dimensional Debye parameter. The results in Fig. 3 are obtained by changing the height of the channel keeping constant the rest of the parameters for the generic flow A. The deceleration effect of EDL results in a decrease of the Nusselt number. The decrease in $N u$ is only a few percent for $\kappa \geq 40$ but may reach $35 \%$ at $\kappa=10$.

\section{Inflexional inviscid instability}

The EDL effect is undoubtedly significant for small values of $\kappa$ for the liquids containing a very small number of for example impurities. In such situations the thickness of the diffuse layer may reach several micrometres. Fig. 2 indicates the close similarity between the EDL and Poiseuille profiles with a decrease of the centreline velocity typical to the EDL flows. The increase of the friction constant is only $16 \%$ in this reference situation. The important difference however is the presence of an inflexional point at $y \approx(1 / \kappa) \operatorname{arcsinh}\left\{-\left(2 / r \kappa^{2}\right) \sinh (\kappa)\right\}$ in the EDL profile where $r$ is the ratio of the EDL and Poiseuille flow centreline velocities. This makes the flow inviscidly unstable, according 
to the Fjortoft's criteria. The inviscid instability does not imply instability directly in wall flows and an Orr-Sommerfeld analysis is necessary.

\section{Linear stability analysis}

The linear hydrodynamic stability under the EDL effect is studied through classical methods. The Orr-Sommerfeld equation is solved by a Galerkin-like procedure [26]. The normal mode solutions of the disturbance equation are:

$$
(u, v, p)=R(\hat{u}, \hat{v}, \hat{p}) \exp (i \alpha x)
$$

$R$ is the real part, $\alpha$ is the dimensionless wave number of the disturbance and $x$ is the streamwise coordinate. Introducing the stream function:

$$
\psi(x, y, t)=\phi(y, t) \exp (i \alpha x)
$$

the Orr-Sommerfeld equation takes the form:

$$
\frac{\partial}{\partial t} L \phi=\frac{1}{R e} L^{2} \phi-i \alpha\left(u L \phi-\frac{\mathrm{d}^{2} u}{\mathrm{~d} y^{2}} \phi\right)
$$

with the boundary conditions $\phi=\partial \phi / \partial y=0$ at $y= \pm 1$. The operator $L$ is $L \equiv$ $\partial^{2} / \partial y^{2}-\alpha^{2}$. The stream function is expanded in a Chebyshev polynomial series:

$$
\phi(y, t)=\sum_{n=1}^{N} a_{n}(t) T_{2 n-2}(y)
$$

where $T_{m}(y)=\cos \left(m \cos ^{-1} y\right)$ denotes the Chebyshev polynomials of the first kind. We took $N=256$ through this study. Eq. (23) takes the form:

$$
\mathbf{Q} \frac{\mathrm{d} \mathbf{a}}{\mathrm{d} t}=(\mathbf{P}-i \alpha \mathbf{J}) \cdot \mathbf{a}
$$

and the matrices are determined by making use of the $\tau$-method described by [16]. The last equation can be written as:

$$
\frac{\mathrm{d} \mathbf{b}}{\mathrm{d} t}=\mathbf{D} \cdot \mathbf{b}
$$

by introducing $\mathbf{B}$ the matrix that diagonalizes $\mathbf{Q}^{-1} \cdot(\mathbf{P}-i \alpha \mathbf{J}), \mathbf{b}=\mathbf{B}^{-\mathbf{1}} \cdot \mathbf{a}$ and the diagonal matrix $\mathbf{D}=\lambda_{i} \delta_{i j}=\mathbf{B}^{-1} \cdot \mathbf{Q}^{-1} \cdot(\mathbf{P}-i \alpha \mathbf{J}) \cdot \mathbf{B}$. The eigenvalues are denoted by $\lambda_{i}$ and the flow is unstable when $R\left(\lambda_{i}\right)>0$. The disturbances with symmetric streamfunctions are considered only. The method gave very close results to Grosch and Salwen [6] who used different sets of expansion functions.

The macroscale Poiseuille flow is metastable; i.e. the corresponding stability is subcritical. Non-linear analysis shows that the instability may occur with finite amplitude when all infinitesimal disturbances are stable $[2,10]$. There is a significantly lower critical value of the Reynolds number $R e_{G}$ compared to $R e_{c}$, above which the flow is unstable and below which there is no bifurcation. The exact theoretical determination of $\operatorname{Re}_{G}$ is 


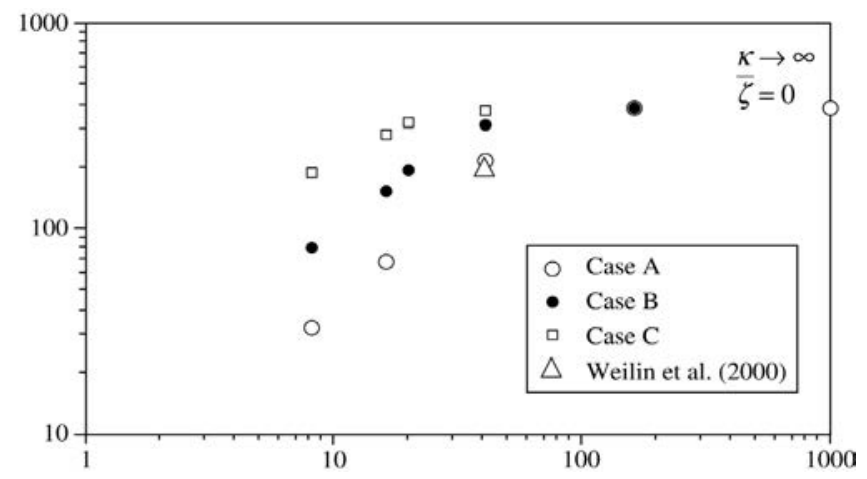

Fig. 4. The critical Reynolds number versus the non-dimensional Debye length under the EDL effect. The reference physical flow parameters for the cases A, B, C and D are given in the text.

still a matter of research. Experiments show that $\operatorname{Re}_{G} / R e_{c} \approx 1000 / 5772 \approx 1 / 6$. The transitional Reynolds number depends on the shape and shape factors of the channels.

The Poiseuille flow is monotonically stable only for $R e<100$ to be compared with 5772. The transitional Reynolds number $R e_{t}$ of channel flows is about 400 according to experiments (2000 based on the hydraulic diameter and channel averaged velocity). Thus, the ratio $R e_{t} / R e_{c}<1 / 15$ in macroscale Poiseuille flows. There are some reasons to believe that this ratio is much smaller under the EDL effect [24]. An estimation of the transitional Reynolds number distribution is shown in Fig. 4 by taking the same ratio $R e_{t} / R e_{c}$ as in Poiseuille flow for the cases A, B and C defined before.

It is clearly seen that the transitional Reynolds number decreases by a factor nearly equal to 2 under the effect of EDL for the case A already at $\kappa=41$. The critical wave and Reynolds numbers of the microflow A are respectively $\alpha_{c}=1.10$ and $R e_{c}=3190$ to be compared with $\alpha_{c}=1.02$ and $R e_{c}=5772$ of the conventional Poiseuille flow $(\bar{\zeta}=0$ or $\kappa \rightarrow \infty$ ). Due to its inviscid inflexional instability (unstable for $\operatorname{Re} \rightarrow \infty$ for a given $\alpha$ ), the band of unstable wave numbers of the EDL micro-flow is significantly larger compared with the macro-flow. The effect disappears quickly when the height of the channel is increased by a factor of 4 . This goes in the same line as previous experimental results showing the lack of micro-effects for the microchannels of height larger than typically $400 \mu \mathrm{m}$. For smaller values of $\kappa$, in return, the effect of the interfacial effects caused by EDL on the transition may be much more severe. For instance, the critical Reynolds number decreases up to $R e_{c}=1042$ at $\kappa=20$ and to $R e_{c}=496$ at $\kappa=10$ for the case A.

Decreasing the parameter $G \bar{\zeta}^{2}$ by a factor of 10 , increases the critical Reynolds number by approximately 3 . Thus, the destabilizing effect of the EDL is much more sensitive to $\kappa$ compared to $G \bar{\zeta}^{2}$. Even at $G=30$ (Case C) the critical (transitional) Reynolds number is twice smaller at $\kappa=8$ than the $R e_{c}$ of the macro-flow. A more complete parametrical study of the EDL effect on the microchannel flow linear stability can be found in [25].

There are only few investigations in the literature on the EDL effect, reaching these Reynolds numbers with relatively small $\kappa$. It is however interesting to note that one point 
related to the data reported by Weilin et al. [28] coincides perfectly with the predicted $R e_{t}$ (profiles at $D_{h} / 2=71 \mu \mathrm{m}$ in their Figure 8) and suggests a transitional number of 200 . That is however certainly a coincidence, because the $G \bar{\zeta}^{2}$ parameter in their investigation is relatively smaller, unless the non-linear EDL stability mechanism is significantly strong.

\section{Preliminary investigation of the by-pass transition under the EDL effect}

There are at least two fundamental reasons to investigate the by-pass transition in microchannel flows. This transition scenario is related to the disturbance growth on time scales significantly shorter than typical Tollmien-Schilichting (TS) waves that "by-passes" the spatial and temporal development of the two-dimensional disturbances and their inherent secondary instabilities. The set-up of three-dimensionality leads to the achievement of finite amplitudes and of the non-linear effects. They can mainly be generated by local surface irregularities such as the roughness or the nanobubbles. The latter is, of course not a micro-effect, because it affects the characteristics in macro- and micro-flows exactly in the same manner at similar scaled roughness dimensions. Yet, it is more difficult to control the roughness geometry and dimensions in microchannels. Note that the roughness does not affect the stability characteristics of a developed internal flow, in the TS waves sense, except in the entrance region. Thus, the by-pass transition process is a presumably more common phenomena in micro-flows. The second reason to investigate the development of the 3D localized disturbances has to do with the associated rapid disturbance algebraic growth. The two-dimensional waves are always the least stable modes according to the Squire's theorem. However, the damping of oblique waves may be smaller at subcritical Reynolds numbers as already shown and mentioned in several investigations (see [8] for example).

The EDL effect on the non-linear stability mechanism is analysed through the spatiotemporal development of a localized disturbance by direct numerical simulations in this investigation. A perturbation related to a pair of counter rotating vortices is followed in time and space with and without EDL. The main aim is to investigate the effect of the double electrostatic layer on the linear, non-linear and breakdown stages in the by-pass transition process. Only some preliminary results are discussed here and more details will be published elsewhere.

The instantaneous 3D non-dimensional Navier Stokes equation under the EDL external force:

$$
\frac{\partial u_{i}}{\partial t}+\frac{\partial u_{i} u_{j}}{\partial x_{j}}=-\frac{\partial p}{\partial x_{i}}+\frac{1}{R e} \frac{\partial^{2} u_{i}}{\partial x_{j}^{2}}-\frac{r \kappa^{2}}{R e} \frac{|\sinh \kappa y|}{\sinh \kappa}
$$

is adequately resolved in time and space. The number of modes is $256 \times 128 \times 128$ in the $x, y$ and $z$ directions. The resolution for the Poiseuille flow is better than [8] for the small perturbation amplitude case. The initial structure is two pairs of counter-rotating vortices exactly as in [8]. The form is:

$$
\psi=\varepsilon f(y)\left(\frac{x^{\prime}}{l_{x}}\right) z^{\prime} \exp \left[-\left(\frac{x^{\prime}}{l_{x}}\right)^{2}-\left(\frac{z^{\prime}}{l_{z}}\right)^{2}\right]
$$


and the velocity components of the perturbed field are:

$$
\left(u^{\prime}, v^{\prime}, w^{\prime}\right)=\left(-\psi_{y} \sin \vartheta, \psi_{z^{\prime}},-\psi_{y} \cos \vartheta\right)
$$

in respectively the streamwise, wall normal and spanwise directions. The lengths $l_{x}$ and $l_{z}$ stand for the streamwise and spanwise lengths of the disturbance $\left(l_{x}=l_{z}=2\right)$ and $\varepsilon$ is its amplitude. The angle $\vartheta$ of the disturbance can be used as a parameter governing the distribution of the initial energy in different regions of wave number space. We took $f(y)=(1+y)^{p}(1-y)^{q}$ with $p=q=2$, as in [8]. The perturbations are superimposed to the mean velocity profile as initial conditions, and the flow field is followed in time and space.

We will present preliminary results with the small (linear) and large (non-linear) perturbation cases corresponding respectively to $\varepsilon=0.0001$ and $\varepsilon=0.1$ in the initial development stage. A flow (case D) with a relatively severe EDL effect is chosen for the purpose and for the same reasons. We took $\kappa=10, G \bar{\zeta}^{2}=600$. There are 30 computational modes in the EDL layer in this case. The critical Reynolds number in the EDL flow is 635. Since the aim is to analyse the subcritical nature of the by-pass mechanism, we took $R e=3000$ in the Poiseuille flow and $R e=300$ in the EDL flow, i.e. ten times smaller than in the macro-flow.

Fig. 5 shows the spatial evolution of the $v^{\prime}$ component of the perturbation velocities $(\vartheta=0)$ at $y=-0.56$ and $t=10$ in Poiseuille and EDL flows in the small perturbation case. We see the characteristics of the dispersion of a wave packet with streamwise elongation and spanwise inclination of the velocity contours. Globally the time and space evolution of the wall normal and spanwise perturbation velocities are both qualitatively and quantitatively similar in the Poiseuille and EDL flows (remember that the EDL Reynolds number is ten times smaller). However, the spanwise gradient of $v^{\prime}$ is significantly more important in EDL than the Poiseuille flow pointing at a relative enhancement of the streamwise vorticity (not shown here). In the non-linear stage the perturbation flow field rapidly develops into a streaky structure under the EDL effect (Fig. 6). These preliminary results show the capacity of the inflexional profile to maintain the non-linear mechanisms even at significantly low Reynolds numbers. Similar behaviour has been found for the $\mathrm{C}$ case at the same $R e / R e_{c}$.

\section{Conclusion}

To conclude, the EDL destabilizes the linear modes of the Poiseuille channel flow and early transition in microchannels is plausible. The most significant effect of EDL is the decrease of the critical Reynolds number, rather than the increase in friction coefficient or the apparent viscosity. For $\kappa=40$, the friction factor increases by some $7 \%$, but the critical Reynolds number DECREASES by $100 \%$.

This effect can be experimentally checked, provided that the liquid contains a very small amount of ions and the channel height is sufficiently small. In practice that would require the use of organic liquids. There is no effect on stability for liquids with high ionic concentration. The non-linear stability analysis of the EDL flow is necessary, although physical considerations and preliminary results presented here on the by-pass transition 

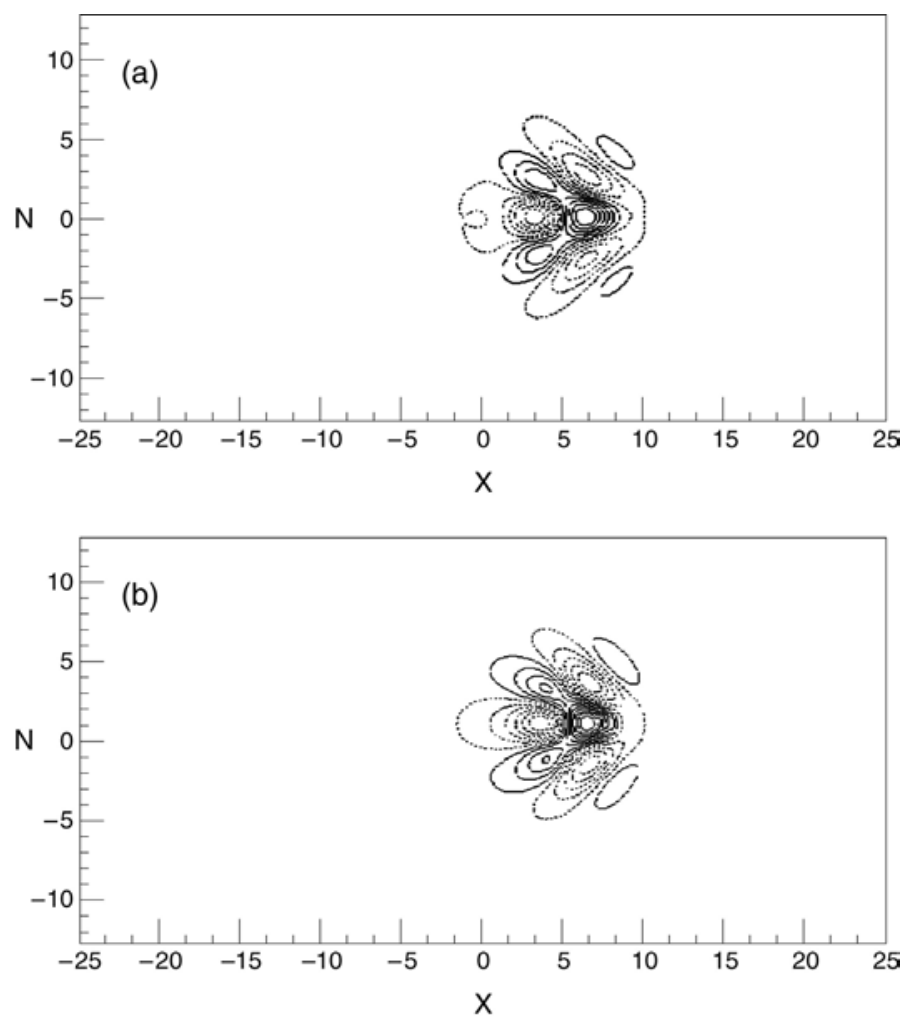

Fig. 5. The wall normal perturbation velocity contours for (a) Poiseuille (Re = 3000) and (b) EDL flows $(R e=300)$ at $t=10$ and $y=-0.56$ in the small amplitude perturbation case. The maximum and minimum non-dimensional velocities in Poiseuille flow are $\pm 5 \times 10^{-6}$, and in EDL flow $\pm 4 \times 10^{-6} . \vartheta=0$.

mechanism indicate a much more rapid transition compared to macro-flows. Controlled experiments in a way similar to those reported by $[20,21]$ have to be conducted, by keeping the same channel with the same roughness distribution, and changing the ionic concentration of the liquid, or of the additives in organic liquids. The fact that the channel cross section shapes, in particular the corners have an important contribution to the EDL field has also to be considered.

There are several open questions concerning the effect of the early transition on the heat and momentum transfer mechanisms [5, 17]. Early transition under the EDL effect is plausible, but the fact that the transitional Reynolds numbers are significantly small raises several points. First of all, the existence of a constant stress universal logarithmic region in near wall flows requires the existence of an inertial sublayer and the Reynolds number to be large. The absence of the logarithmic region, on the other hand, implies the absence of inertial subrange of energy spectra and consequently the equilibrium between production and dissipation. Thus, the well-known velocity defect law and the core region structure are presumably quite different from macro-scale turbulent channel flow. Neither any 

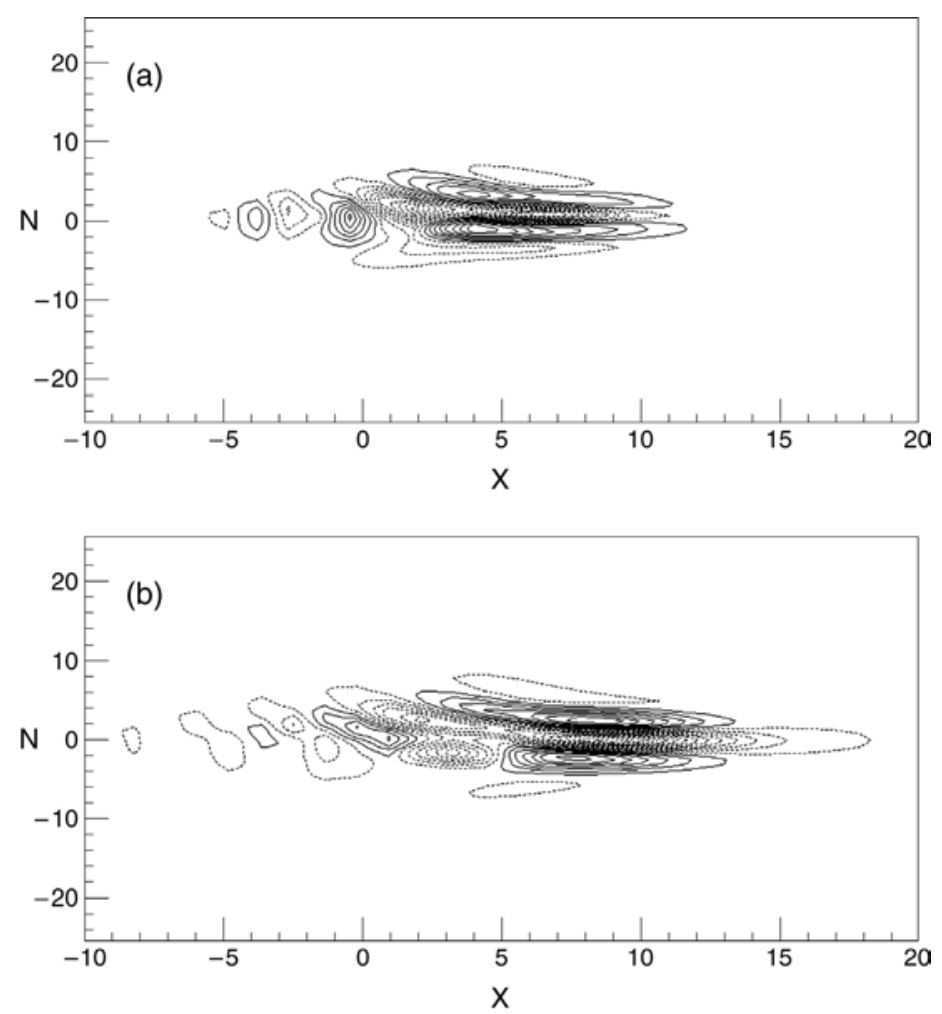

Fig. 6. The wall normal (a) and spanwise (b) perturbation velocity contours for the EDL flow $(R e=300)$ at $t=15$ and $y=-0.56$ in the large amplitude perturbation case. The maximum and minimum non-dimensional velocities are $\pm 7 \times 10^{-3}$ in (a) and $\pm 2 \times 10^{-2}$ in (b). $\vartheta=\pi / 4$.

well-known logarithmic friction law, nor the analogies such as Prandtl-Taylor's can be used in this case. These observations open interesting perspectives in near wall turbulence research.

\section{References}

[1] S. Andriamitanjo, Electrisation d'un hydrocarbure s'écoulant dans un tube métallique en régime laminaire, Thèse de l'INPG, Grenoble, France, 1981.

[2] P.G. Drazin, W.H. Reid, Hydrodynamic stability, Cambridge University Press, Cambridge, 1981, pp. 370-464 (Chapter 7).

[3] D. Erickson, D. Li, C. Werner, An improved method of determining the Zeta potential and surface conductance, J. Colloid Interface Sci. 232 (2000) 186-197.

[4] M. Gad-el-Hak, The fluid mechanics of microdevices, J. Fluids Eng. 121 (1999) 5-33.

[5] P. Gao, S. Le Person, M. Favre Marinet, Scale effects on hydrodynamics and heat transfer in twodimensional mini and microchannels, Int. J. Thermal Sci. 41 (2002) 10.

[6] C.E. Grosch, H. Salwen, The stability of steady and time-dependent plane Poiseuille flow, J. Fluid Mech. 34 (1968) 177-205. 
[7] F. Gui, R.P. Scaringe, Enhanced heat transfer in the entrance region of microchannels, in: Proceedings of the 30th Intersociety Eng. Conf., vol. 2, 1995, pp. 284-294.

[8] D.S. Henningson, A. Lundbladh, A. Johansson, A mechanism for bypass transition from localized disturbances in wall-bounded shear flows, J. Fluid Mech. 250 (1993) 169-207.

[9] R.J. Hunter, Zeta Potential in Colloid Science, Academic Press, London, 1981.

[10] N. Itoh, Spatial growth of finite wave disturbances in parallel and nearly parallel flows. Part 1. The theoretical analysis and the numerical results for plane Poiseuille flow, Trans. Japan Soc. Aeronant. Space Sci. 17 (1974) 160-174.

[11] L. Kulinsky, Y. Wang, M. Ferrari, Electroviscous effects in microchannels, in: Proceedings of SPIE, vol. 3606, The International Society of Optical Engineering, 1999, pp. 158-168.

[12] D. Li, Electrokinetic effects on pressure-driven liquid flow in microchannels, in: A.V. Delgado (Ed.), Interfacial Electrokinetics and Electrophoresis, Marcel Dekker Inc., New York, 2002.

[13] J. Lykelma, M. Minor, Colloids Surf. A 140 (33) (1997) 1.

[14] G.M. Mala, D. Li, J.D. Dale, Heat transfer and fluid flow in microchannels, Int. J. Heat Mass Transfer 40 (13) (1997) 3079-3088.

[15] G.M. Mala, D. Li, Flow characteristics of water in microtubes, Int. J. Heat Fluid Flow 20 (1999) 142-148.

[16] S.A. Orszag, Accurate solution of Orr-Sommerfield stability equation, J. Fluid Mech. 50 (1971) 689-703.

[17] X.F. Peng, G.P. Peterson, B.X. Wang, Heat transfer characteristics of water flowing through microchannels, Exp. Heat transfer 7 (1994) 265-283.

[18] M.M. Rahman, F. Gui, Experimental measurements of fluid flow and heat transfer in microchannel cooling passages in a chip substrate, ASME EEP 4 (2) (1993) 685-692.

[19] M.M. Rahman, F. Gui, Design, fabrication, and testing microchannel heat sinks for aircraft avionics cooling, in: Proceedings of the 28th Intersociety Energy Conversion Eng. Conf., 1993, pp. 1-6.

[20] L. Ren, D. Li, W. Qu, Electro-viscous effects on liquid flow in microchannels, J. Colloid Interface Sci. 233 (2001) 12-22.

[21] L. Ren, W. Qu, D. Li, Interfacial electrokinetic effects on liquid flow in microchannels, Int. J. Heat Mass Transfer 44 (2001) 3125-3134.

[22] K.V. Sharp, R.J. Adrian, J.G. Santiago, J.I. Molho, Liquid flows in microchannels, in: M. Gad-el-Hak (Ed.), The MEMS Handbook, CRC Press, LLC, 2002.

[23] S. Tardu, Transferts thermiques dans les microcanaux, in: Microfluidique, Traité EGEM, Tome 6, Hermès, 2004, p. 36 (Chapitre 6).

[24] S. Tardu, Interfacial electrokinetic effects on microchannel flow linear stability, J. Fluid Eng. 126 (2004) $10-13$.

[25] S. Tardu, Analysis of the electric double layer effect on the microchannel flow stability, Int. J. Microscale Thermophys. Eng. (in press).

[26] C.H. Von Kerczek, The instability of oscillatory Poiseuille flow, J. Fluid Mech. 116 (1982) 91-114.

[27] B.X. Wang, X.F. Peng, Experimental investigation on liquid forced convection heat transfer through microchannels, Int. J. Heat Mass Transfer 37 (1994) 73-82.

[28] Q. Weilin, G.M. Mala, L. Dongqing, Pressure-driven water flows in trapezoidal silicon microchannels, Int. J. Heat Mass Transfer 43 (2000) 353-364.

[29] C. Yang, D. Li, J.H. Masliyah, Modelling forced liquid convection in rectangular microchannels with electrokinetic effects, Int. J. Heat Mass Transfer 41 (1998) 4229-4249.

[30] J. Yang, F. Lu, L. Kostiuk, D. Kwok, Electrokinetic microchannel battery by means of electrokinetic and microfluidic phenomena, J. Micromech. Microeng. 13 (2003) 963-970. 